\title{
Spin-orbit effects in structural and electronic properties for the solid state of the group-14 elements from carbon to superheavy element 114
}

\author{
Andreas Hermann* \\ Department of Chemistry, The University of Auckland, Auckland, New Zealand and MacDiarmid Institute for Advanced Materials \\ and Nanotechnology, School of Chemical and Physical Sciences, Victoria University, Wellington, New Zealand \\ Jürgen Furthmüller \\ Institut für Festkörpertheorie und -optik, Friedrich-Schiller-Universität Jena, Max-Wien-Platz 1, D-07743 Jena, Germany \\ Heinz W. Gäggeler ${ }^{\dagger}$ and Peter Schwerdtfeger \\ Centre for Theoretical Chemistry and Physics, The New Zealand Institute for Advanced Study, \\ Massey University (Auckland Campus), Private Bag 102904, North Shore City, 0745 Auckland, New Zealand
}

(Received 6 August 2010; published 8 October 2010)

\begin{abstract}
Spin-orbit effects approximately scale like $Z^{2}$ and therefore become very important in the bonding of the heavier $p$-group elements in the periodic table. Here we show by first-principles density-functional calculations that such effects substantially lower the cohesive energy for solid lead and Uuq (ununquadium, eka-lead, nuclear charge 114), by $2.5 \mathrm{eV} /$ atom for the latter and causing a structural change from face-centered cubic at the scalar relativistic to hexagonal close packed at the spin-orbit coupled level of theory. This implies that unlike lead (cohesive energy $E_{c o h}=2.02 \mathrm{eV} /$ atom), Uuq is weakly bound $\left(E_{c o h}=0.5 \mathrm{eV} /\right.$ atom), and even less so than solid mercury $\left(E_{c o h}=0.7 \mathrm{eV} /\right.$ atom $)$, underpinning the original hypothesis by Pitzer in $1975[\mathrm{~K}$. Pitzer, J. Chem. Phys. 63, 1033 (1975)] that spin-orbit effects lead to chemical inertness of Uuq.
\end{abstract}

DOI: 10.1103/PhysRevB.82.155116

PACS number(s): 31.15.aj, 31.15.am, 31.15.es, 31.15.vn

\section{INTRODUCTION}

It is now well established that relativistic effects cannot be neglected in solid-state properties of heavy element containing compounds. ${ }^{1-11}$ This is known since Christensen and Seraphin published their seminal paper on relativistic effects in the band structure of solid gold. ${ }^{1}$ In fact, Loucks pointed out as early as 1966 that for tungsten experimental results are in better agreement with relativistic augmented plane-wave calculations than the nonrelativistic (NR) ones. ${ }^{12}$ Scalar relativistic effects are responsible for the valence $s$ contraction (direct relativistic effect) and the expansion of the $d$ and $f$ shells (indirect relativistic effect) through the increased nuclear screening by the direct relativistic effects. ${ }^{13,14}$ However, for heavy elements spin-orbit (SO) effects need to be considered as well, as they increase roughly with $Z^{2}(Z$ being the nuclear charge). In fact, the atomic spin-orbit stabilization of the $p_{1 / 2}$ shell leads to a significant reduction in stability for thallium and lead compounds, ${ }^{13,15-21}$ and the importance of spin-orbit effects has already been recognized in 1965 for properties in solid lead by Anderson ${ }^{22}$ and Loucks. ${ }^{23}$ Concerning the element following lead in group 14, element 114 (ununquadium, abbreviated as Uuq for the following), Pitzer suggested in 1975 that Uuq should be chemically inert ${ }^{24}$ and could even be a gas at room temperature. This is in accordance with recent calculations on the static electric polarizability for the ${ }^{3} \mathrm{P}_{0}$ state of atomic Uuq, which, except for carbon, is the lowest among the group-14 elements, ${ }^{25,26}$ and for the electron affinity of Uuq which is predicted to be the smallest (close or equal to zero) in this series of elements. ${ }^{27}$

With the recent discovery of superheavy elements up to atomic number 118 (Uuo), ${ }^{28,29}$ and the independent confir- mation of some of those elements, ${ }^{30,31}$ it became possible to investigate their chemical properties. Uuq is of special interest: this member of the periodic table is predicted to be in the center of the island of superheavy elements, stabilized by nuclear shell effects. ${ }^{32}$ Isotopes of Uuq with sufficiently long half lives required for chemical study $\left(T_{1 / 2} \geq 0.5 \mathrm{~s}\right)$ are ${ }^{287} \operatorname{Uuq}\left(T_{1 / 2}=0.5 \mathrm{~s}\right)$, formed in the reaction between ${ }^{48} \mathrm{Ca}$ projectiles and a ${ }^{242} \mathrm{Pu}$ target, ${ }^{288} \mathrm{Uuq}\left(T_{1 / 2}=0.8 \mathrm{~s}\right)$ and ${ }^{289} \operatorname{Uuq}\left(T_{1 / 2}=2.4 \mathrm{~s}\right)$, both formed with the same projectile and a ${ }^{244} \mathrm{Pu}$ target, respectively. First chemical experiments have been conducted with element Uuq, yielding indication for an unexpected high volatility, much higher than that of its homolog in the periodic table $\mathrm{Pb}$ and being between those of $\mathrm{Hg}$ and $\mathrm{Rn} .{ }^{33}$ Element Uuq behaved quite similar to $\mathrm{Cn}$ (element 112), studied earlier. ${ }^{34,35}$ Here we mention that the chemistry of Uuq has already been studied theoretically at the atomic ${ }^{36}$ and molecular level by several research groups, ${ }^{37-44}$ and it has been demonstrated only very recently that the solid state of superheavy element $\mathrm{Cn}$ is influenced greatly by both scalar-relativistic (SR) and spin-orbit effects. ${ }^{11}$ Reviews on superheavy element chemistry can be found in Refs. 45-48.

Spin-orbit effects in the group-14 elemental solids have not been studied in great detail. In a recent paper, Noffsinger and Cohen ${ }^{49}$ predicted solid Uuq to be metallic, that it crystallizes in a face-centered cubic (fcc) structure, and that it behaves much like a dense variant of $\mathrm{Pb}$. However, they concentrated on structural properties and did not give cohesive energies for $\mathrm{Pb}$ or Uuq, thus making it difficult to verify Pitzer's original assumption. ${ }^{24}$ They also neglected spin-orbit effects which could lower the cohesive energy substantially due to the atomic spin-orbit stabilization of the ${ }^{3} \mathrm{P}_{0}\left(7 p_{1 / 2}^{2}\right)$ state, hence potentially leading to significant structural 
TABLE I. Lattice constants $a_{0}$ (in $\AA$ ), nearest-neighbor distance $r_{\text {min }}$ (in $\AA$ ), cohesive energies $E_{\text {coh }}$ (in eV/atom), bulk moduli $B$ (in kbar), and pressure derivatives $B^{\prime}$ of the group-14 elements from DFT-PW91 calculations, and compared to experimental data.

\begin{tabular}{|c|c|c|c|c|c|c|c|c|c|c|c|c|c|c|c|c|}
\hline \multirow[b]{2}{*}{ Element } & \multirow[b]{2}{*}{ Crystal } & $a_{0}$ & $r_{\text {min }}$ & $E_{c o h}$ & $B$ & $B^{\prime}$ & \multirow[b]{2}{*}{ Crystal } & $a_{0}$ & $r_{\min }$ & $E_{c o h}$ & $B$ & $B^{\prime}$ & $a_{0}$ & $r_{\min }$ & $E_{c o h}$ & $B$ \\
\hline & & \multicolumn{5}{|c|}{ SR-PW91 } & & \multicolumn{5}{|c|}{ SO-PW91 } & \multicolumn{4}{|c|}{ Expt. $^{a}$} \\
\hline $\mathrm{C}$ & dia & 3.566 & 1.544 & 7.765 & 4432.2 & 3.9 & dia & 3.566 & 1.544 & 7.776 & 4438.7 & 3.9 & 3.567 & 1.544 & 7.43 & 4422.7 \\
\hline $\mathrm{Si}$ & dia & 5.468 & 2.368 & 4.643 & 884.8 & 4.3 & dia & 5.468 & 2.368 & 4.664 & 884.8 & 4.3 & 5.431 & 2.352 & 4.66 & 978.0 \\
\hline $\mathrm{Ge}$ & dia & 5.758 & 2.493 & 3.828 & 599.9 & 4.8 & dia & 5.758 & 2.493 & 3.771 & 598.9 & 4.8 & 5.658 & 2.450 & 3.86 & 752.0 \\
\hline $\mathrm{Sn}$ & dia & 6.644 & 2.877 & 3.260 & 369.2 & 5.0 & dia & 6.647 & 2.878 & 3.064 & 365.8 & 5.0 & 6.489 & 2.810 & 3.12 & 425.3 \\
\hline $\mathrm{Pb}$ & fcc & 5.039 & 3.563 & 3.012 & 404.8 & 4.8 & fcc & 4.897 & 3.463 & 1.979 & 374.8 & 5.0 & 4.950 & 3.500 & 2.02 & 438.7 \\
\hline Uuq & fcc & 5.326 & 3.766 & 3.021 & 330.6 & 4.9 & hcp & $4.126^{\mathrm{b}}$ & 3.834 & 0.504 & 145.7 & 5.8 & & & & \\
\hline
\end{tabular}

${ }^{\mathrm{a}}$ Experimental values from Refs. 59 and 60.

${ }^{\mathrm{b}}$ For the hep structure of Uuq the lattice constant $c_{0}$ is $6.014 \AA$

changes. We therefore decided to extend on Noffsinger and Cohen's work and study spin-orbit effects in solid-state properties for the whole group-14 series from carbon down to Uuq in the periodic table.

\section{COMPUTATIONAL DETAILS}

Structural and electronic properties of the group-14 elements were calculated using density-functional theory (DFT) as implemented in the Vienna $a b$ initio simulation package (VASP). ${ }^{50}$ The electronic exchange-correlation energy was approximated using the Perdew-Wang (PW91) and PerdewBurke-Ernzerhof (PBE) parametrizations ${ }^{51,52}$ within the projector augmented wave (PAW) method to describe the electron-ion interactions. ${ }^{53,54} \mathrm{~A}$ plane-wave energy cutoff of $E_{c}=340 \mathrm{eV}$ was employed throughout all calculations, and sufficiently large regular $k$-point meshes $^{55}$ were used to sample the Brillouin zone.

All standard PAW data sets used here are constructed from scalar-relativistic all-electron calculations. Thus, scalarrelativistic effects leading to contraction of the inner $s$ electron shells, and expansion of the outer $d$ - and $f$-electron shells, are inherently included in all DFT calculations. To assess the impact of relativity on the heavier elements' crystalline properties, we also constructed PAW data sets for $\mathrm{Pb}$ and Uuq from nonrelativistic all-electron calculations. All PAW valence spaces are of the form $(n-1) d n(s p)$, thus including four valence electrons $(\mathrm{C}, \mathrm{Si})$ and 14 valence electrons per atom ( $\mathrm{Ge}, \mathrm{Sn}, \mathrm{Pb}$, and $\mathrm{Uuq}$ ) in our calculations, respectively, and allowing for small polarizations of the $(n-1) d$ core. The $5 f$ states of Uuq are located about 5 Ry below the $6 d$ and $7 s$ states, and are therefore considered as strongly bound core states, in contrast to the approach by Noffsinger and Cohen. This allows for using the same moderate plane-wave cutoff throughout the whole group. We also performed spin-orbit calculations for all elements of group 14, which proved crucial in obtaining the correct crystal structures and cohesive energies for the heavy elements $\mathrm{Pb}$ and Uuq. As implemented in VASP, spin-orbit contributions are only calculated on the radial grids inside the PAW spheres. Since the contributions to the spin-orbit matrix elements are strongest close to the nuclei, the cutoff radii (which increase from 1.5 a.u. for $\mathrm{C}$ to 2.5 a.u. for $\mathrm{Pb}$ and 3.0 a.u. for Uuq) are not expected to influence the results significantly.

For all elements, the energy-volume relation $E(V)$ was obtained over a range of lattice constants for a variety of crystal classes: fcc, body-centered cubic (bcc), hexagonal close packed (hcp), simple cubic (sc), and diamond (dia). Ground-state properties such as lattice constants, cohesive energies, and bulk moduli were then obtained from equationof-state fits. ${ }^{56}$ For hcp structures, the $c / a$ ratio of the lattice constants was optimized over a range of unit-cell volumes.

Electronic band structures and densities of state (DOSs) were calculated at the respective optimized geometries. Converged DOS results were obtained using much denser $k$-point grids and the tetrahedron method ${ }^{57}$ was used to interpolate between $k$ points. The same $k$-point grid was used to obtain Fermi-surface plots for the metallic states of $\mathrm{Pb}$ and Uuq, which were visualized using the XCRYSDEN program. ${ }^{58}$

\section{RESULTS}

The results obtained for the most stable crystal structures are given in Table I comparing both SR and SO results with experimental data. Carbon, silicon, germanium, and tin all adopt the diamond crystal structure ${ }^{61}$ in perfect agreement with our DFT calculations. Christensen et al. ${ }^{14}$ already pointed out that at the nonrelativistic level $\mathrm{Pb}$ adopts the diamond structure as well, which is also in agreement with our results shown in Table II. In fact, if relativistic effects are completely ignored, both $\mathrm{Pb}$ and Uuq crystallize in the diamond structure - the low-temperature crystal phase found for all lighter elements in group 14. When including scalarrelativistic effects (but still ignoring spin-orbit coupling), both $\mathrm{Pb}$ and Uuq prefer to crystallize in the fcc structure, which is slightly favored over both the hcp and bcc crystal structures, cf. Table II. This is in agreement with the findings of Christensen et al. for $\mathrm{Pb}$ and with Noffsinger and Cohen ${ }^{49}$ for both $\mathrm{Pb}$ and Uuq. A different picture emerges, however, if spin-orbit coupling is included; while $\mathrm{Pb}$ still prefers to crystallize in the fcc structure (with a low-pressure transition to hcp, see below), Uuq then crystallizes in the hcp crystal structure (with a transition to fcc under pressure, see below). 
TABLE II. Lattice constants (in $\AA$ ), nearest-neighbor distance $r_{\text {min }}$ (in $\AA$ ), cohesive energies $E_{\text {coh }}$ (in eV/atom), and bulk moduli $B$ (in kbar) for different lattice symmetries of $\mathrm{Pb}$ and Uuq from DFT-PW91 calculations.

\begin{tabular}{|c|c|c|c|c|c|c|c|c|c|c|c|c|c|}
\hline \multirow[b]{2}{*}{ Element } & \multirow[b]{2}{*}{ Crystal } & $a_{0}$ & $r_{\min }$ & $E_{c o h}$ & $B$ & $a_{0}$ & $r_{\min }$ & $E_{c o h}$ & $B$ & $a_{0}$ & $r_{\min }$ & $E_{c o h}$ & $B$ \\
\hline & & \multicolumn{4}{|c|}{ DFT-GGA-NR } & \multicolumn{4}{|c|}{ DFT-GGA-SR } & \multicolumn{4}{|c|}{ DFT-GGA-SO } \\
\hline \multirow[t]{5}{*}{$\mathrm{Pb}$} & dia & 7.073 & 3.063 & 3.215 & 329.2 & 7.051 & 3.053 & 2.762 & 259.1 & 7.097 & 3.073 & 1.807 & 228.3 \\
\hline & $\mathrm{fcc}$ & 5.100 & 3.606 & 3.072 & 425.2 & 5.039 & 3.563 & 3.012 & 404.8 & 4.897 & 3.463 & 1.979 & 374.8 \\
\hline & hcp ${ }^{a}$ & 3.578 & 3.595 & 3.086 & 434.7 & 3.549 & 3.559 & 2.996 & 399.6 & 3.506 & 3.574 & 1.966 & 368.3 \\
\hline & bcc & 4.025 & 3.485 & 3.083 & 437.3 & 3.995 & 3.459 & 2.969 & 395.0 & 4.009 & 3.472 & 1.951 & 364.0 \\
\hline & $\mathrm{sc}$ & 3.269 & 3.269 & 3.131 & 430.1 & 3.251 & 3.251 & 2.833 & 362.8 & 3.267 & 3.267 & 1.871 & 325.7 \\
\hline \multirow[t]{5}{*}{ Uuq } & dia & 7.718 & 3.342 & 2.928 & 259.4 & 7.578 & 3.281 & 2.351 & 184.5 & 8.029 & 3.477 & 0.397 & 62.9 \\
\hline & fcc & 5.014 & 3.546 & 2.867 & 353.6 & 5.326 & 3.766 & 3.021 & 330.6 & 5.511 & 3.897 & 0.487 & 124.8 \\
\hline & $h c p^{a}$ & 3.848 & 3.867 & 2.888 & 369.0 & 3.720 & 3.765 & 2.996 & 330.8 & 4.126 & 3.834 & 0.504 & 145.7 \\
\hline & bcc & 4.327 & 3.747 & 2.887 & 374.5 & 4.209 & 3.645 & 2.959 & 324.4 & 4.375 & 3.789 & 0.486 & 128.2 \\
\hline & $\mathrm{sc}$ & 3.540 & 3.540 & 2.905 & 354.6 & 3.460 & 3.460 & 2.626 & 278.2 & 3.641 & 3.641 & 0.444 & 94.0 \\
\hline
\end{tabular}

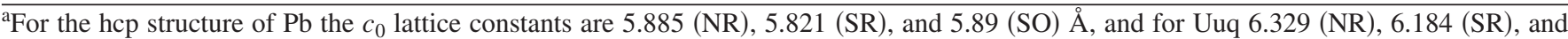
6.014 (SO) A.

The decrease in Pb's fcc lattice constant caused by spin-orbit interactions is about $3 \%$, significantly larger than the $0.2 \%$ change found in a recent density-functional perturbationtheory study of Pb's phonon spectrum. ${ }^{62}$ The reason for this difference is not immediately clear but is probably related to the different pseudopotential methods used (PAW vs norm conserving).

Within the group, advancing from the light to the heavy elements, there is a monotonous trend toward larger nearestneighbor distances, smaller cohesive energies, and smaller bulk moduli as one expects for the $p$-group elements. The nearest-neighbor distances are in very good agreement with available crystal structure measurements but slightly overestimated due to the DFT approximation used. Note that it is currently not trivial to obtain better agreement with experiment by using DFT-generalized gradient approximation (GGA), and $a b$ initio incremental methods using coupled cluster techniques ${ }^{63-68}$ or the recently developed randomphase approximation ${ }^{69-71}$ are far more computer time demanding, and it remains to be seen how these methods perform for strongly correlated metallic systems. The slight overestimations in the nearest-neighbor distance results in an overestimation of the unit-cell volumes (the volumes per atom from our spin-orbit calculations are in cubic angstrom: C 5.669, Si 20.438, Ge 23.866, Sn 36.703, Pb 32.164, and Uuq 48.339). Accordingly, mass densities (here given in grams per cubic centimeter) are too low when compared to experiment (experimental values are in parentheses): C 3.518 (3.52), Si 2.282 (2.33), Ge 5.054 (5.33), Sn 5.371 (5.77), Pb 10.697 (11.35), and Uuq 9.928. Here we used the standard molar masses for all elements, except for Uuq where we used $289 \mathrm{~g} / \mathrm{mol}$ for isotope ${ }^{289} \mathrm{Uuq}$. Note that Uuq is thus predicted to have a lower mass density than $\mathrm{Pb}$, even though the element is about $40 \%$ heavier. The spin-orbit hep structure of Uuq also exhibits an unusually low $c / a$ ratio of $1.458(\mathrm{~Pb}$ : $1.680)$, much lower than the ideal value of 1.633 , and also significantly lower than the scalar-relativistic value $c / a$ $=1.663(\mathrm{~Pb}: 1.640)$. Such a "compression" of the hexagonal layers is intriguing, also since it is in contrast to the unusu- ally high $c / a$ values found in group-12 elements $\mathrm{Zn}$ and Cd. ${ }^{72}$

In Fig. 1, the cohesive energies across the group-14 elements (while neglecting zero-point vibrational effects) are compared to experimental standard heats of formation. Here, the PW91 exchange-correlation functional used for most of the present calculations is also compared to the PBE functional for the elements $\mathrm{C}$ through $\mathrm{Pb}$. The graph illustrates the importance of spin-orbit effects for the heavier elements, which become much more relevant than scalar-relativistic effects for $\mathrm{Pb}$ and Uuq. The cohesive energy of $\mathrm{Pb}$ is reduced by spin-orbit effects by about $1 \mathrm{eV}$ to $1.98 \mathrm{eV} /$ atom, and

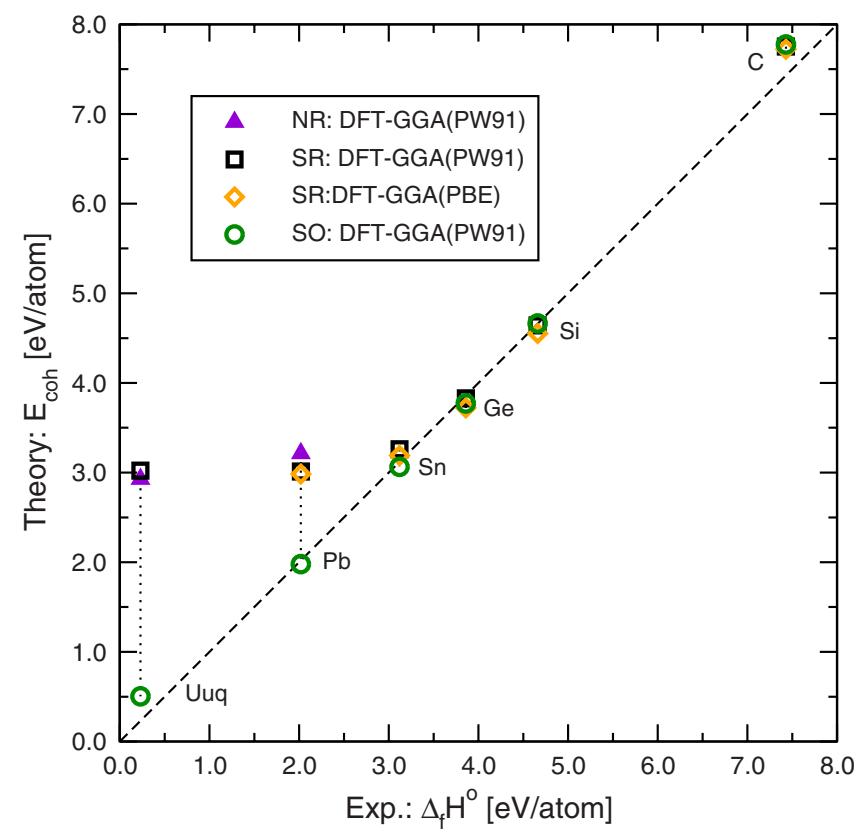

FIG. 1. (Color online) Cohesive energies of group-14 elementary crystals, comparing various theoretical methods, plotted against experimental heats of formation (Ref. 60). For Uuq, the experimental value of $0.23 \mathrm{eV}$ is based on its adsorption enthalpy on gold (Refs. 33 and 73). 
$\mathrm{Pb}$

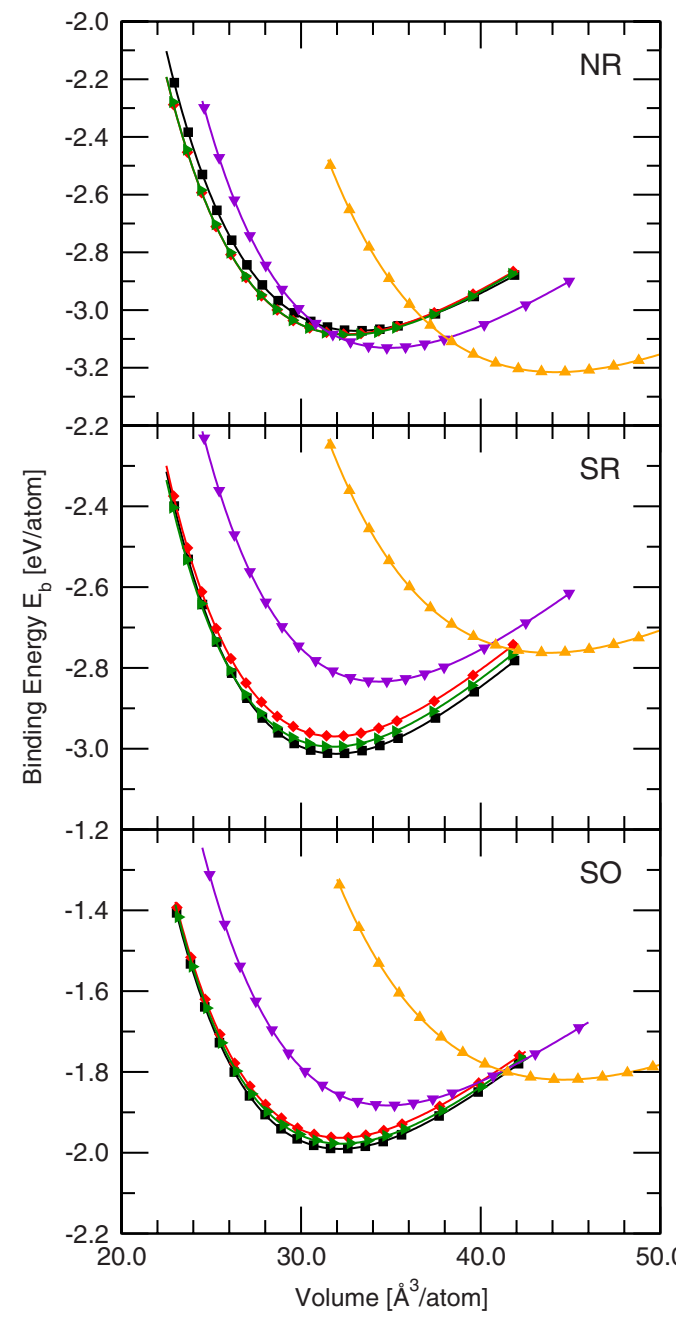

Uuq

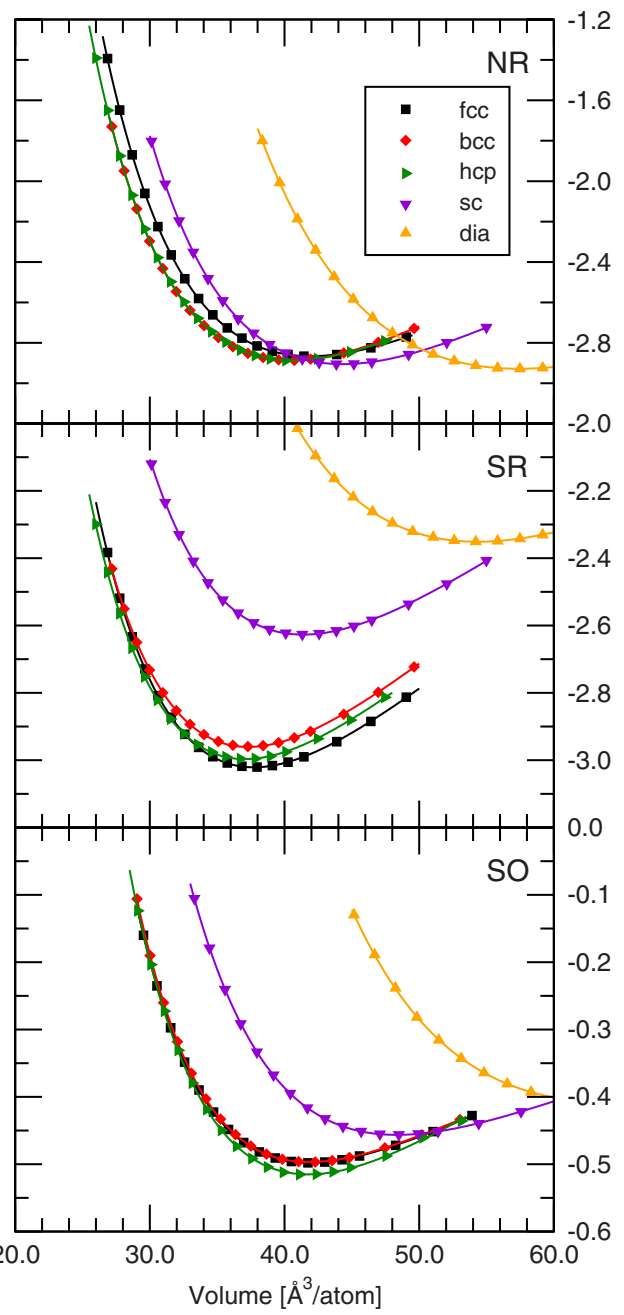

FIG. 2. (Color online) Binding energies of Pb (left) and Uuq (right) as function of unit-cell volume. From top: NR, SR, and SO results. Data points (symbols) and equation-of-state fits (solid lines) are shown.

Uuq's cohesive energy is decreased by about $2.5 \mathrm{eV}$ to 0.50 $\mathrm{eV} / \mathrm{atom}$. This brings the cohesive energy of $\mathrm{Pb}$ very close to the experimental value of $2.02 \mathrm{eV}^{60}$ The spin-orbit interaction lowers the energy of the free $\mathrm{Pb}$ atom by $1.60 \mathrm{eV}$, and the lattice energy of fcc- $\mathrm{Pb}$ by $0.56 \mathrm{eV}$ per atom, resulting in a net decrease in the binding energy. From these results we expect that Uuq is far more volatile than $\mathrm{Pb}$. Its calculated cohesive energy of $0.50 \mathrm{eV}$ is also in very good agreement with a recent estimate by Pershina et al. ${ }^{44}$ of about $0.7 \mathrm{eV}$, based on dimer dissociation energies. We mention that the spin-orbit contributions to the cohesive energy approximately follow the trend $\Delta_{s o} E_{c o h}=1.81 \times 10^{-4} \mathrm{eV} \times Z^{2}$ with larger deviations for the lighter elements which show rather small spin-orbit effects. The slight spin-orbit-induced stabilization of the diamond structures of carbon and silicon is difficult to explain and could be an artifact of our DFT approximation.

Plots of the binding energy (negative of the cohesive energy) of $\mathrm{Pb}$ and Uuq crystals versus volume of the unit cell are shown in Fig. 2, comparing the different Hamiltonians (nonrelativistic: NR, scalar relativistic: SR; and spin-orbit:
SO) as well as the different crystal structures investigated. We note that Noffsinger and Cohen find the ordering $E_{b}(\mathrm{fcc})<E_{b}(\mathrm{bcc})<E_{b}(\mathrm{hcp})$ for Uuq at the scalar-relativistic level, whereas we find at the same level of theory $E_{b}(\mathrm{fcc})$ $<E_{b}$ (hcp) $<E_{b}$ (bcc). The dia and sc structures are much less favored compared to the nonrelativistic case. The influence of scalar relativity on the cohesive energies of both $\mathrm{Pb}$ and Uuq is minor, both exhibit a cohesive energy of about 3 eV/atom, see Table II.

In high-pressure experiments, $\mathrm{Pb}$ is found to initially undergo a phase transition from fcc to a hcp structure at about $13 \mathrm{GPa}$, and then to a bcc structure, starting at about $90 \mathrm{GPa}$ but with a large hysteresis due to the small volume difference between the hcp and bcc structures. ${ }^{74-76}$ We studied the pressure dependence of both $\mathrm{Pb}$ and Uuq by evaluating the Gibbs free energy at low temperatures, $G=E+p V$, for all crystal structures, based on the ground-state parameters in Table II. In a nonrelativistic world, both $\mathrm{Pb}$ and Uuq would follow the structural path $\mathrm{dia} \rightarrow \mathrm{sc} \rightarrow(\mathrm{hcp} / \mathrm{bcc})$, where the latter two structures are almost indistinguishable for both elements, see also Fig. 2. The transition pressures are $1.5 \mathrm{GPa}(0.3 \mathrm{GPa})$ 

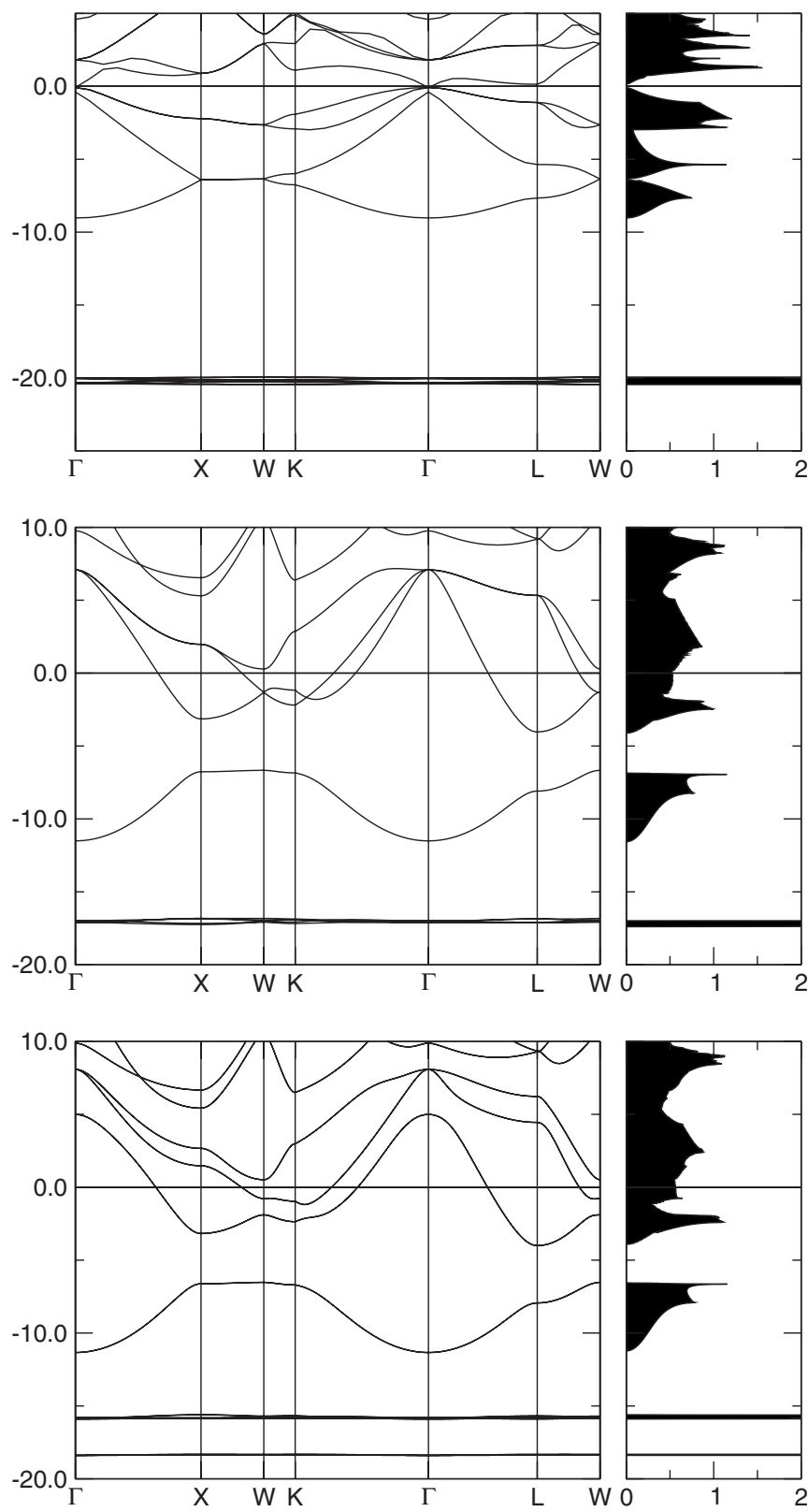
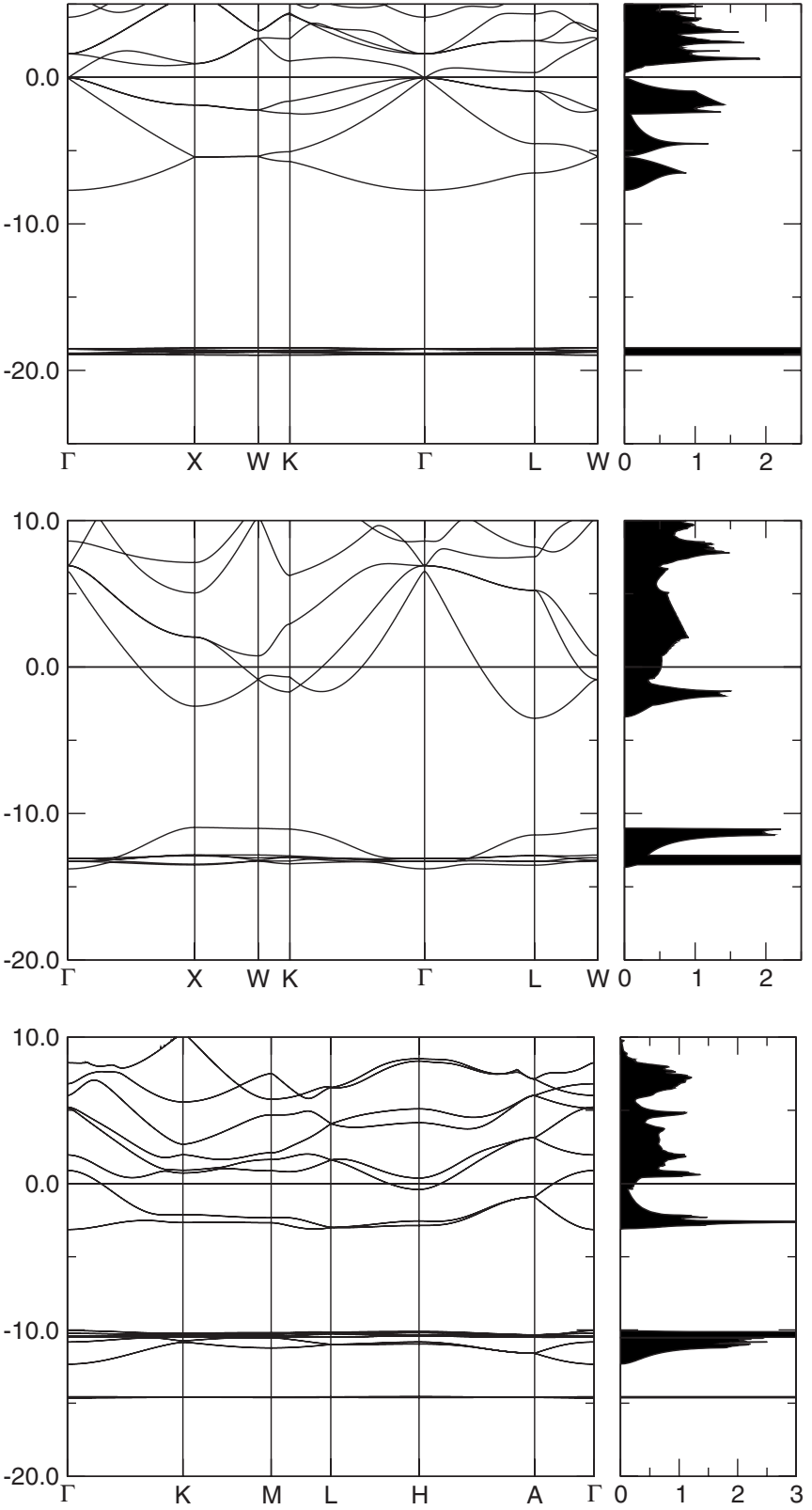

FIG. 3. Electronic band structures and DOSs, for both Pb (left) and Uuq (right). From top to bottom: NR (both diamond structures), SR (both fcc structures), and SO (Pb: fcc; Uuq: hcp). All energies with respect to the Fermi level; DOS in states per electron volt per atom.

for $\mathrm{dia} \rightarrow \mathrm{sc}$ and $3.3 \mathrm{GPa}(0.8 \mathrm{GPa})$ for $\mathrm{sc} \rightarrow(\mathrm{hcp} / \mathrm{bcc})$, for $\mathrm{Pb}$ (Uuq). In a scalar-relativistic regime, $\mathrm{Pb}$ follows a fcc $\rightarrow$ hcp $\rightarrow$ bcc structural path, in qualitative agreement with the experimental findings. The transition pressures are 17.9 $\mathrm{GPa}$ for the $\mathrm{fcc} \rightarrow$ hcp transition and $359 \mathrm{GPa}$ for the hcp $\rightarrow$ bcc transition. The first transition pressure agrees well with experiment, however, the transition pressure to the bcc phase is significantly overestimated. Both phases are very close in energy over a wide pressure range, and it is difficult to assign a precise value for the transition pressure. For Uuq, we also find a fcc $\rightarrow$ hcp phase transition, with a transition pressure of $6.3 \mathrm{GPa}$. When including spin-orbit interactions for $\mathrm{Pb}$, we retain the $\mathrm{fcc} \rightarrow \mathrm{hcp} \rightarrow$ bcc structural path, with transition pressures of $29.9 \mathrm{GPa}$ and $69.6 \mathrm{GPa}$, respectively. Both pressures agree qualitatively with the experimental data. For Uuq however, we obtain a significant stabilization of the hcp phase due to spin-orbit coupling, and thus no pressure-induced transitions to other crystal structures are found.

The bulk moduli are also compared in Table II. These are experimentally better accessible than $0 \mathrm{~K}$ cohesive energies and the theoretical results are in reasonable agreement with experiment. The calculated bulk moduli do, however, underestimate the experimental values systematically (except for carbon) by about $10-15 \%$. Including spin-orbit effects generally does not improve the agreement with experiment. All experimental data are obtained near room temperature at ambient pressure; the $0 \mathrm{~K}$ bulk moduli are thus expected to be even larger than the data presented here. It is currently not easy to pin point the exact reason for such a deviation but it could easily originate from the DFT approximation used. 
At all levels of theory, both $\mathrm{Pb}$ and Uuq are found to be metals or semimetals. In Fig. 3, electronic band structures and DOSs are compiled for the optimized crystal structures of both elements, on NR, SR, and SO level of theory. In a nonrelativistic world, both $\mathrm{Pb}$ and Uuq would be diamondlike semimetals, with very low density of states at the Fermi level. The $n s$ and $n p$ valence electrons would form a continuous band with a total width of about $9 \mathrm{eV}$ for $\mathrm{Pb}$ and 8 $\mathrm{eV}$ for Uuq. Including scalar-relativistic effects changes the preferred crystal structures for both elements to fcc and both are metallic. The $n s$ valence bands are separated from the $n p$ bands, and much more stabilized for Uuq than for $\mathrm{Pb}$. Additionally, the unoccupied Uuq $8 s$ band is lowered such that it mixes with the top of the $7 p$ band, at about $7 \mathrm{eV}$ above the Fermi level (note that for element 118 the strong relativistic $8 s$ stabilization leads to a positive electron affinity ${ }^{77}$ ). The Uuq $6 d$ bands are destabilized compared to $\mathrm{Pb} 5 d$, leading to considerable mixing of $6 d$ and $7 s$ in Uuq. Around the Fermi level, however, both elements show similar properties. This is in agreement with the results presented by Noffsinger and Cohen. ${ }^{49}$ Including spin-orbit interactions brings little change for the $\mathrm{DOS}$ of $\mathrm{Pb}$. The spin-orbit splitting between $6 p_{3 / 2}$ and $6 p_{1 / 2}$ is found to be $\Delta_{s o}=4.1 \mathrm{eV}$, and between $5 d_{5 / 2}$ and $5 d_{3 / 2}$ to be $\Delta_{s o}^{\prime}=2.5 \mathrm{eV}$, the latter in rather good agreement with the $5 d^{9} 6 s^{2}{ }^{2} D_{5 / 2} / 2 D_{3 / 2}$ splitting in $\mathrm{Pb}^{3+}(2.64$ eV) ${ }^{78}$ The largest splitting in the $6 p$ levels in atomic lead is between the ${ }^{3} P_{0}$ and ${ }^{1} S_{0}$ with $3.65 \mathrm{eV} \cdot{ }^{78}$ For Uuq, spin-orbit interactions will lead to a change in crystal structure, from fcc to hcp. The band splitting between $6 d_{5 / 2}$ and $6 d_{3 / 2}$ is found to be about $\Delta_{s o}^{\prime}=4.2 \mathrm{eV}$. From the fcc electronic band structure (not shown here) we find a rather large splitting between the $7 p_{3 / 2}$ and $7 p_{1 / 2}$ states of $\Delta_{s o}=7.0 \mathrm{eV}$, but in agreement with previous atomic calculations which show equally large spin-orbit effects..$^{26,45}$

The Fermi surfaces of the metallic phases of $\mathrm{Pb}$ and Uuq, as obtained from scalar-relativistic and spin-orbit calculations, are shown in Fig. 4. For Pb, the two sheeted Fermi surface displays the well-known "jungle gym" topology with a localized closed sheet at the zone center and tubes along the zone edges. The influence of spin-orbit effects on the Fermi surface is small, and results compare well with experimental data and previous spin-orbit calculations. ${ }^{22}$ For fccUuq at the scalar-relativistic level of theory, the Fermi surface is qualitatively very similar to $\mathrm{Pb}$, albeit with a smaller sheet in the zone center and more slender tubes, in agreement with the findings by Noffsinger and Cohen. Spin-orbit effects, however, have a much more pronounced effect on fccUuq than on $\mathrm{Pb}$ : the zone center sheet is much smaller and the tube network along the zone edges is reduced to disjointed sheets centered around the $\mathrm{K}$ and $\mathrm{U}$ points. This ef-
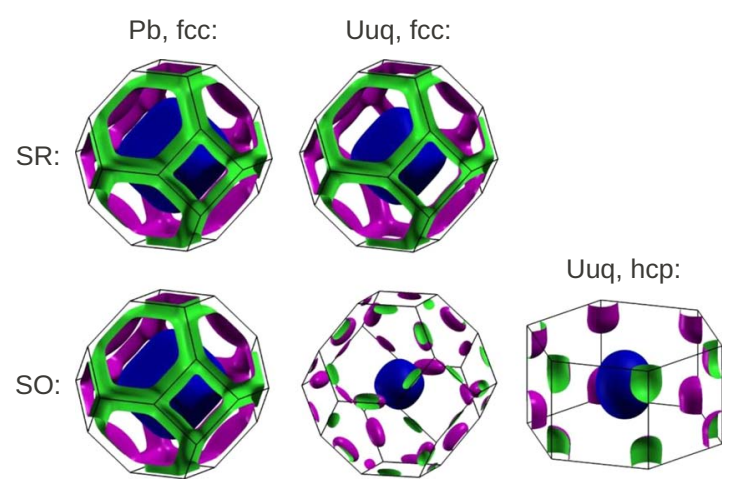

FIG. 4. (Color online) Fermi surfaces for $\mathrm{Pb}$ and Uuq, on scalarrelativistic (upper panel) and spin-orbit level of theory (lower panel). For Uuq, both fcc and hep Fermi surfaces from spin-orbit calculations are shown.

fect originates in the large spin-orbit splitting of $\Delta_{s o}$ $=7.0 \mathrm{eV}$, which lowers the $7 p_{1 / 2}$ band such that the Fermi energy level is only $1.1 \mathrm{eV}$ below the $7 p_{1 / 2}$ band maximum. In the preferred hcp structure, Uuq has one sheet in the zone center and another sheet localized around the $\mathrm{H}$ point.

\section{CONCLUSION}

We demonstrated that spin-orbit effects substantially lower the cohesive energy for solid lead and Uuq, and also lead to a structural phase transition for the latter which adopts the hep phase under normal conditions. The rather low cohesive energy for Uuq $(0.5 \mathrm{eV})$ is in good agreement with Pershina's estimate ${ }^{44}$ or the predicted low sublimation energy of $0.23(+0.22)(-0.08)$ eV by Eichler et al ${ }^{33,73}$ Here we note that for the dimer of Uuq, $\mathrm{Uuq}_{2}$, Liu et al. ${ }^{39}$ have calculated a dissociation energy of only $0.12 \mathrm{eV}$ compared to $2.16 \mathrm{eV}$ at the scalar-relativistic level. However, in contrast to these gas phase results ${ }^{39}$ we find an increase in the nearest-neighbor distance due to spin-orbit effects, thus the large destabilization outweighs the contraction of the $7 p_{1 / 2}$ shell. We predict that solid Uuq is less stable than solid $\mathrm{Pb}$ and perhaps less stable than solid $\mathrm{Hg}$, supporting the original hypothesis by Pitzer in 1975 (Ref. 24) that spin-orbit effects lead to a large increase in chemical inertness for Uuq.

\section{ACKNOWLEDGMENTS}

Financial support by the Royal Society of New Zealand through a Marsden Grant and by the MacDiarmid Institute is gratefully acknowledged. A.H. wants to thank N. W. Ashcroft for fruitful discussions.

\footnotetext{
*a.hermann@auckland.ac.nz

$\dagger$ On leave from the Paul Scherrer Institut, CH-5232 Villigen PSI, Switzerland; heinz.gaeggeler@psi.ch

*p.a.schwerdtfeger@massey.ac.nz

${ }^{1}$ N. E. Christensen and B. O. Seraphin, Phys. Rev. B 4, 3321 (1971).
}

\footnotetext{
${ }^{2}$ N. Takeuchi, C. T. Chan, and K. M. Ho, Phys. Rev. B 40, 1565 (1989).

${ }^{3}$ C. Elsässer, N. Takeuchi, K. M. Ho, C. T. Chan, P. Braun, and M. Fähnle, J. Phys.: Condens. Matter 2, 4371 (1990).

${ }^{4}$ P. P. Singh, Phys. Rev. B 49, 4954 (1994).

${ }^{5}$ T. Söhnel, H. Hermann, and P. Schwerdtfeger, Angew. Chem.,
} 
Int. Ed. 40, 4381 (2001).

${ }^{6}$ N. E. Christensen, in Relativistic Electronic Structure Theory. Part 1: Fundamentals, edited by P. Schwerdtfeger (Elsevier, Amsterdam, 2002), p. 863.

${ }^{7}$ H. Eschrig, M. Richter, and I. Opahle, in Relativistic Electronic Structure Theory. Part 2: Applications, edited by P. Schwerdtfeger (Elsevier, Amsterdam, 2004), p. 724.

${ }^{8}$ T. Söhnel, H. Hermann, and P. Schwerdtfeger, J. Phys. Chem. B 109, 526 (2005).

${ }^{9}$ N. Gaston, B. Paulus, K. Rosciszewski, P. Schwerdtfeger, and H. Stoll, Phys. Rev. B 74, 094102 (2006).

${ }^{10}$ N. Gaston and P. Schwerdtfeger, Phys. Rev. B 74, 024105 (2006).

${ }^{11}$ N. Gaston, I. Opahle, H. W. Gäggeler, and P. Schwerdtfeger, Angew. Chem., Int. Ed. 46, 1663 (2007).

${ }^{12}$ T. L. Loucks, Phys. Rev. 143, 506 (1966)

${ }^{13}$ P. Pyykko, Chem. Rev. 88, 563 (1988).

${ }^{14}$ N. E. Christensen, S. Satpathy, and Z. Pawlowska, Phys. Rev. B 36, 1032 (1987).

${ }^{15}$ H. Overhof and J. Treusch, Solid State Commun. 9, 53 (1971).

${ }^{16}$ P. Schwerdtfeger, Phys. Scr. 36, 453 (1987).

${ }^{17}$ P. Schwerdtfeger, H. Silberbach, and B. Miehlich, J. Chem. Phys. 90, 762 (1989).

${ }^{18}$ P. Schwerdtfeger, G. A. Heath, M. Dolg, and M. A. Bennett, J. Am. Chem. Soc. 114, 7518 (1992).

${ }^{19}$ K. K. Das, H.-P. Liebermann, R. J. Buenker, and G. Hirsch, J. Chem. Phys. 104, 6631 (1996).

${ }^{20}$ Y.-K. Han and K. Hirao, J. Chem. Phys. 112, 9353 (2000).

${ }^{21}$ M. Iliaš, V. Kellö, L. Visscher, and B. Schimmelpfennig, J. Chem. Phys. 115, 9667 (2001).

${ }^{22}$ J. R. Anderson and A. V. Gold, Phys. Rev. 139, A1459 (1965).

${ }^{23}$ T. L. Loucks, Phys. Rev. Lett. 14, 1072 (1965).

${ }^{24}$ K. S. Pitzer, J. Chem. Phys. 63, 1033 (1975).

${ }^{25}$ C. Thierfelder, B. Assadollahzadeh, P. Schwerdtfeger, S. Schäfer, and R. Schäfer, Phys. Rev. A 78, 052506 (2008).

${ }^{26}$ V. Pershina, A. Borschevsky, E. Eliav, and U. Kaldor, J. Chem. Phys. 128, 024707 (2008).

${ }^{27}$ A. Borschevsky, V. Pershina, E. Eliav, and U. Kaldor, Chem. Phys. Lett. 480, 49 (2009).

${ }^{28}$ Yu. Ts. Oganessian, J. Phys. G 34, R165 (2007).

${ }^{29}$ Yu. Ts. Oganessian, F. Sh. Abdullin, P. D. Bailey, D. E. Benker, M. E. Bennett, S. N. Dmitriev, J. G. Ezold, J. H. Hamilton, R. A. Henderson, M. G. Itkis, Yu. V. Lobanov, A. N. Mezentsev, K. J. Moody, S. L. Nelson, A. N. Polyakov, C. E. Porter, A. V. Ramayya, F. D. Riley, J. B. Roberto, M. A. Ryabinin, K. P. Rykaczewski, R. N. Sagaidak, D. A. Shaughnessy, I. V. Shirokovsky, M. A. Stoyer, V. G. Subbotin, R. Sudowe, A. M. Sukhov, Yu. S. Tsyganov, V. K. Utyonkov, A. A. Voinov, G. K. Vostokin, and P. A. Wilk, Phys. Rev. Lett. 104, 142502 (2010).

${ }^{30}$ L. Stavsetra, K. E. Gregorich, J. Dvorak, P. A. Ellison, I. Dragojević, M. A. Garcia, and H. Nitsche, Phys. Rev. Lett. 103, 132502 (2009).

${ }^{31}$ Ch. E. Düllmann, M. Schädel, A. Yakushev, A. Türler, K. Eberhardt, J. V. Kratz, D. Ackermann, L.-L. Andersson, M. Block, W. Brüchle, J. Dvorak, H. G. Essel, P. A. Ellison, J. Even, J. M. Gates, A. Gorshkov, R. Graeger, K. E. Gregorich, W. Hartmann, R.-D. Herzberg, F. P. Heßberger, D. Hild, A. Hübner, E. Jäger, J. Khuyagbaatar, B. Kindler, J. Krier, N. Kurz, S. Lahiri, D. Liebe, B. Lommel, M. Maiti, H. Nitsche, J. P. Omtvedt, E. Parr, D. Rudolph, J. Runke, B. Schausten, E. Schimpf, A. Semchenkov,
J. Steiner, P. Thörle-Pospiech, J. Uusitalo, M. Wegrzecki, and N. Wiehl, Phys. Rev. Lett. 104, 252701 (2010).

${ }^{32}$ W. D. Myers and W. J. Swiatecki, Nucl. Phys. 81, 1 (1966).

${ }^{33}$ R. Eichler, N. V. Aksenov, Yu. V. Albin, A. V. Belozerov, G. A Bozhikov, V. I. Chepigin, S. N. Dmitriev, R. Dressler, H. W. Gäggeler, V. A. Gorshkov, R. A. Henderson, A. M. Johnsen, J. M. Kenneally, V. Ya. Lebedev, O. N. Malyshev, K. J. Moody, Yu. Ts. Oganessian, O. V. Petrushkin, D. Piguet, A. G. Popeko, P. Rasmussen, A. Serov, D. A. Shaughnessy, S. V. Shishkin, A. V. Shutov, M. A. Stoyer, N. J. Stoyer, A. I. Svirikhin, E. E. Tereshatov, G. K. Vostokin, M. Wegrzecki, P. A. Wilk, D. Wittwer, and A. V. Yeremin, Radiochim. Acta 98, 133 (2010).

${ }^{34}$ R. Eichler, N. V. Aksenov, A. V. Belozerov, G. A. Bozhikov, V. I. Chepigin, S. N. Dmitriev, R. Dressler, H. W. Gäggeler, V. A. Gorshkov, F. Haenssler, M. G. Itkis, A. Laube, V. Ya. Lebedev, O. N. Malyshev, Yu. Ts. Oganessian, O. V. Petrushkin, D. Piguet, P. Rasmussen, S. V. Shishkin, A. V. Shutov, A. I. Svirikhin, E. E. Tereshatov, G. K. Vostokin, M. Wegrzecki, and A. V. Yeremin, Nature (London) 447, 72 (2007).

${ }^{35}$ R. Eichler, N. V. Aksenov, A. V. Belozerov, G. A. Bozhikov, V. I. Chepigin, S. N. Dmitriev, R. Dressler, H. W. Gäggeler, A. V. Gorshkov, M. G. Itkis, F. Haenssler, A. Laube, V. Ya. Lebedev, O. N. Malyshev, Yu. Ts. Oganessian, O. V. Petrushkin, D. Piguet, A. G. Popeko, P. Rasmussen, S. V. Shishkin, A. A. Serov, A. V. Shutov, A. I. Svirikhin, E. E. Tereshatov, G. K. Vostokin, M. Wegrzecki, and A. V. Yeremin, Angew. Chem., Int. Ed. 47, 3262 (2008).

${ }^{36}$ A. Landau, E. Eliav, Y. Ishikawa, and U. Kaldor, J. Chem. Phys. 114, 2977 (2001).

${ }^{37}$ M. Seth, K. Faegri, and P. Schwerdtfeger, Angew. Chem., Int. Ed. Engl. 37, 2493 (1998).

${ }^{38}$ C. S. Nash and B. E. Bursten, J. Phys. Chem. A 103, 402 (1999).

${ }^{39}$ W. Liu, C. Van Wüllen, Y.-K. Han, and Y.-S. Lee, Adv. Quantum Chem. 39, 325 (2001).

${ }^{40}$ P. Schwerdtfeger and M. Seth, J. Nucl. Radiochem. Sci. 3, 133 (2002).

${ }^{41}$ A. V. Zaitsevskii, C. van Wüllen, and A. V. Titov, Russ. Chem. Rev. 78, 1173 (2009)

${ }^{42}$ V. Pershina, J. Anton, and T. Jacob, J. Chem. Phys. 131, 084713 (2009).

${ }^{43}$ C. Gourlaouen, O. Parisel, and J.-P. Piquemal, Chem. Phys. Lett. 469, 38 (2009).

${ }^{44}$ V. Pershina, A. Borschevsky, J. Anton, and T. Jacob, J. Chem. Phys. 132, 194314 (2010).

${ }^{45} \mathrm{P}$. Schwerdtfeger and M. Seth, in Encyclopedia of Computational Chemistry, edited by P. von R. Schleyer, P. R. Schreiner, N. L. Allinger, T. Clark, J. Gasteiger, P. A. Kollman, and H. F. Schaefer, III (Wiley, New York, 1998), Vol. 4, p. 2480.

${ }^{46}$ V. Pershina, in Relativistic Electronic Structure Theory, Part 2, edited by P. Schwerdtfeger (Elsevier, New York, 2004), p. 1.

${ }^{47}$ Superheavy Elements, edited by M. Schädel (Kluwer, Dordrecht, 2003).

${ }^{48}$ M. Schädel, Angew. Chem., Int. Ed. 45, 368 (2005).

${ }^{49}$ J. Noffsinger and M. L. Cohen, Phys. Rev. B 81, 073110 (2010).

${ }^{50}$ G. Kresse and J. Furthmüller, Phys. Rev. B 54, 11169 (1996).

${ }^{51}$ J. P. Perdew, J. A. Chevary, S. H. Vosko, K. A. Jackson, M. R. Pederson, D. J. Singh, and C. Fiolhais, Phys. Rev. B 46, 6671 (1992).

${ }^{52}$ J. P. Perdew, K. Burke, and M. Ernzerhof, Phys. Rev. Lett. 77, 3865 (1996). 
${ }^{53}$ P. E. Blöchl, Phys. Rev. B 50, 17953 (1994).

${ }^{54}$ G. Kresse and D. Joubert, Phys. Rev. B 59, 1758 (1999).

${ }^{55}$ H. J. Monkhorst and J. D. Pack, Phys. Rev. B 13, 5188 (1976).

${ }^{56}$ F. Birch, Phys. Rev. 71, 809 (1947).

${ }^{57}$ P. E. Blöchl, O. Jepsen, and O. K. Andersen, Phys. Rev. B 49, 16223 (1994).

${ }^{58}$ A. Kokalj, J. Mol. Graphics Modell. 17, 176 (1999).

${ }^{59}$ Springer Handbook of Condensed Matter and Materials Data, edited by W. Martienssen and H. Warlimont (Springer, Berlin, 2005).

${ }^{60}$ J. D. Cox, D. D. Wagman, and V. A. Medvedev, CODATA Key Values for Thermodynamics (Hemisphere, New York, 1989).

${ }^{61}$ N. W. Ashcroft and N. D. Mermin, Solid State Physics (Thomson Learning, USA, 1976).

${ }^{62}$ M. J. Verstraete, M. Torrent, F. Jollet, G. Zérah, and X. Gonze, Phys. Rev. B 78, 045119 (2008).

${ }^{63}$ B. Paulus, K. Rościszewski, N. Gaston, P. Schwerdtfeger, and H. Stoll, Phys. Rev. B 70, 165106 (2004).

${ }^{64}$ H. Stoll, B. Paulus, and P. Fulde, J. Chem. Phys. 123, 144108 (2005).

${ }^{65}$ E. Voloshina, B. Paulus, and H. Stoll, J. Phys.: Conf. Ser. 117, 012029 (2008).
${ }^{66}$ A. Hermann and P. Schwerdtfeger, Phys. Rev. Lett. 101, 183005 (2008).

${ }^{67}$ H. Stoll, B. Paulus, and P. Fulde, Chem. Phys. Lett. 469, 90 (2009).

${ }^{68}$ H. Stoll, J. Phys. Chem. A 113, 11483 (2009).

${ }^{69}$ J. Harl and G. Kresse, Phys. Rev. B 77, 045136 (2008).

${ }^{70}$ J. Harl and G. Kresse, Phys. Rev. Lett. 103, 056401 (2009).

${ }^{71}$ B. G. Janesko, T. M. Henderson, and G. E. Scuseria, J. Chem. Phys. 130, 081105 (2009).

${ }^{72}$ N. Gaston and B. Paulus, Phys. Rev. B 76, 214116 (2007).

${ }^{73}$ R. Eichler, Radiochim. Acta 93, 245 (2005).

${ }^{74}$ T. Takahashi, H. K. Mao, and W. A. Bassett, Science 165, 1352 (1969).

${ }^{75}$ H. Mao, Y. Wu, J. Shu, J. Hu, R. Hemley, and D. Cox, Solid State Commun. 74, 1027 (1990).

${ }^{76}$ C. A. Vanderborgh, Y. K. Vohra, H. Xia, and A. L. Ruoff, Phys. Rev. B 41, 7338 (1990).

${ }^{77}$ E. Eliav, U. Kaldor, Y. Ishikawa, and P. Pyykkö, Phys. Rev. Lett. 77, 5350 (1996).

${ }^{78}$ C. E. Moore, Atomic Energy Levels, Natl. Bur. Stand. (U.S.) Circ. No. 467 (U.S. GPO Washington, DC, 1958). 